\title{
有序介孔硅/碳复合结构负极材料的制备与电化学性能研究
}

\author{
王 静 $^{1}$, 陈志柠 ${ }^{1}$, 郭玉忠 ${ }^{1}$, 黄瑞安 ${ }^{2}$, 王剑华 ${ }^{1}$ \\ (昆明理工大学 1. 材料科学与工程学院; 2. 真空冶金国家工程实验室, 昆明 650093)
}

摘 要: 以 SBA-15 $\left(\mathrm{SiO}_{2}\right)$ 有序介孔模板为前驱体, 通过镁热还原和碳包覆修饰路线, 制备出二维定向组装的束捆状 有序介孔覆碳纳米组织(OMP-Si/C), 并细致研究了 SBA-15 的镁热还原过程, 对 OMP-Si/C 复合结构进行电化学性 能测试。经 XRD 测试分析发现: 镁热还原过程中存在 $\mathrm{Mg}_{2} \mathrm{Si}$ 中间相反应路径, 基于此提出反应温度-时间 $(T-t)$ 相 转变图; DSC/TG 表明, $\mathrm{Mg}$ 相通过反应一熔化协调联动作用, 在低于熔点 $\left(648^{\circ} \mathrm{C}\right)$ 时熔化, 且发生液/固反应。从 FE-SEM 观察到 SBA-15 柱状单元被装配成类藕根链束捆状 Si 有序介孔组织, 并基于镁液微浴流场卷吸作用机理解 释了该二维定向组装过程。所得致密纳米组织能有效抵消充放电过程中 Si 相的固有体积变化, 从而表现出优异的 循环稳定性能和倍率性能。

关 键 词: 锂离子电池; $\mathrm{Si}$ 有序介孔组织; 镁热还原反应; $\mathrm{Mg}_{2} \mathrm{Si}$ 中间相; 微流场装配 中图分类号: 0646 文献标识码: A

\section{Preparation and Electrochemical Performance of Ordered Mesoporous Si/C Composite for Anode Material}

\author{
WANG Jing ${ }^{1}$, CHENG Zhi-Ning ${ }^{1}$, GUO Yu-Zhong ${ }^{1}$, HUANG Rui-An², WANG Jian-Hua ${ }^{1}$
}

(1. Faculty of Materials Science and Engineering, Kunming University of Science and Technology, Kunming 650093, China; 2. National Engineering Laboratory for Vacuum Metallurgy, Kunming University of Science and Technology, Kunming 650093, China)

\begin{abstract}
Using SBA-15 of $\mathrm{SiO}_{2}$ template as precursor, a bundle-shaped ordered mesoporous nanoarchitecture $\mathrm{Si} / \mathrm{C}$ composite (OMP-Si/C) was successfully assembled 2D-directionally by a magnesiothermic reduction reaction (MRR) route and the ensuing carbon-coating modification processing, and the MRR process of the SBA-15 was investigated in depth, the electrochemical performance of as-prepared OMP-Si/C composites was tested. Analysis based on XRD data reveals the presence of a reaction path of $\mathrm{Mg}_{2} \mathrm{Si}$ intermediate phase in MRR process, on which a $T-t$ (reaction temperature-reaction time) phase transition diagram is proposed. DSC/TG analysis shows that Mg particles may melt below its melting point $\left(648^{\circ} \mathrm{C}\right)$ upon reaction, and in liquid-solid reaction pattern react with $\mathrm{SiO}_{2}$ via melting-reaction teamwork. Observed from FE-SEM, SBA-15 column units are validated to assemble into a lotus-root-chain-bundle shaped nanoarchitecture of ordered mesoporous silicon, which can effectively offset the drastic volume change of Si material inherent in the charge/discharge process, and exhibit excellent cycling stability and rate capability. Based on the micro-fluid field assembling mechanism, the two-dimensional directional assembly process can be reasonably interpreted.
\end{abstract}

Key words: Li-ion battery; ordered mesoporous Si architecture; magnesiothermic reduction reaction; intermediate phase of $\mathrm{Mg}_{2} \mathrm{Si}$; micro fluid field assembly

收稿日期：2017-03-30; 收到修改稿日期：2017-06-14

基金项目：国家自然科学基金(51464025) National Natural Science Foundation of China (51464025)

作者简介：王 静(1992-), 女, 硕士研究生. E-mail: 1456429099@qq.com

通讯作者：郭玉忠，教授.E-mail: yzguocn62@sina.com; 黄瑞安，副教授.E-mail: rahuang2002@163.com 
硅材料以其高达 $3600 \mathrm{mAh} / \mathrm{g}$ 的理论比容量、原 料丰富易得、环境友好等特点, 有望取代商用石墨 作为下一代负极材料 ${ }^{[1-3]}$ 。然而, 硅材料存在一系列 技术障碍并未实现商业化应用: (1)在嵌/脱 $\mathrm{Li}$ 过程 中会产生原 Si 颗粒 3 倍以上的体积胀缩, 导致微米 级 Si 颗粒碎裂、组织坍塌、活性物质与集电极间 剥离等电极失效问题, 表现为电池容量的急剧衰 减 ${ }^{[4-5]}$; (2) 在循环过程中巨大体积变化进一步诱致 固体电解质界面(SEI)不稳定并不断消耗 $\mathrm{Li}^{+}$和电解 质, 容量进一步衰减 ${ }^{[6]}$ 。为解决 $\mathrm{Si}$ 体积膨胀问题, 研 究者们近年来提出纳米结构化路线, 即将微米初 级单元硅设计制备成各种纳米尺寸结构, 如硅纳 米线 ${ }^{[7]} 、$ 纳米管 ${ }^{[8]}$ 、纳米空心球 ${ }^{[9]}$ 、纳米片、带、 膜 ${ }^{[10-11]}$ 等。这些纳米单元被直接装配于集电极馢片 上, 形成不易剥落的整体式纳米电极, 其内部空穴、 通道能有效缓冲 $\mathrm{Si}$ 单元的体积效应问题 ${ }^{[12]}$ 。或者, 通过化学刻蚀微米级 Si 颗粒制备各种 Si 介孔组织, 替代前述 $\mathrm{Si}$ 纳米结构 ${ }^{[13-14]}$ 。介孔孔道为 $\mathrm{Li}^{+}$提供快 捷传输通道的同时, 也能有效缓解体膨胀, 使电化 学性能有效提升 ${ }^{[6,13-15]}$ 。另一途径是进行表面包覆修 饰, 引入如金属、碳、氧化物、有机导电高分子等 第二组元 ${ }^{[16]}$, 在改善和强化 Si 纳米构架的机械韧性 和导电性方面效果显著 ${ }^{[17]}$ 。纳米结构化以及表面修 饰有效解决了电极体积效应、结构粉化及 SEI 层不 稳定等问题。然而, 纳米结构极低的堆砌密度会衍 生出储能密度低的问题 ${ }^{[18]}$, 且制备工艺精细复杂、 难以低成本、规模化生产 ${ }^{[19]}$ 。

镁热还原 $\mathrm{SiO}_{2}$ 可在保持原 $\mathrm{SiO}_{2}$ 初始结构不变 下低成本规模化制备的硅纳米结构, 为 $\mathrm{Si}$ 纳米化提 供一个重要途径 ${ }^{[20-21]}$ 。YANG 等 ${ }^{[22]}$ 镁蒸汽还原 SBA-15 分子篮模板, 制备出类莲藕根状硅有序介 孔覆碳材料。利用此线路以相应 $\mathrm{SiO}_{2}$ 结构为原料, 已成功制备出来硅纳米球 ${ }^{[7]}$ 、纳米管 ${ }^{[8]}$ 、纳米线 ${ }^{[9]}$ 。 但是, $\mathrm{SiO}_{2}$ 镁热还原过程较为复杂, 其动力学路径、 产物相组成及空间分布等与其原料组织、混料均匀 性、加热制度等众多因素密切相关 ${ }^{[23]}$ 。其中镁热还 原产物中是否出现 $\mathrm{Mg}_{2} \mathrm{Si}$ 等副产物, 会直接影响 $\mathrm{Si}$ 收率的高低, 进而对电化学性能产生影响。

本工作以 SBA-15 分子篮为前驱体经镁热还原 制备 $\mathrm{Si}$ 有序介孔覆碳复合纳米结构, 细致研究了 $\mathrm{SiO}_{2}$ 还原过程中物相组成、动力学途径及 $\mathrm{Si}$ 介孔结 构变化趋势, 并对样品的电化学循环行为进行表征, 以期获得较高 Si 收率和对初始结构单元堆砌装配 方式的深入理解。

\section{1 实验方法}

\section{1 材料制备}

将市购的介孔二氧化硅 SBA-15(南京先锋纳米 有限公司, AR, 99\%)与镁粉(200 目, 中国国药集团 化学试剂有限公司, AR, 99\%)按 1:1 的质量比, 机 械混匀后转移至刚玉舟中, 在氩气保护的管式炉中 以 $4^{\circ} \mathrm{C} / \mathrm{min}$ 升至预定温度下保温预定时间。随炉冷 却后, 反应产物经 $1 \mathrm{~mol} / \mathrm{L} \mathrm{HCl}$ 浸洗后用去离子水、 无水乙醇清洗后, 经 $60^{\circ} \mathrm{C}$ 真空干燥 $12 \mathrm{~h}$, 即得到有 序介孔硅样品(标记为 OMP-Si)。利用庶糖热解法对 OMP-Si 表面进行碳包覆修饰, 获得碳包覆样品(标 记为 $\mathrm{OMP}-\mathrm{Si} / \mathrm{C})$, 利用氧化失重法 $\left(700^{\circ} \mathrm{C}\right)$ 分析样品 包碳量为 $23 \%$, 其工艺操作见文献 ${ }^{[24]}$ 。

\section{2 材料测试分析}

使用 Rigaku III 型 X 射线衍射(XRD)仪对材料 进行物相分析, CuK $\alpha 1$ 线 $(0.15406 \mathrm{~nm})$, 管电压 $40 \mathrm{kV}$, 管电流 $100 \mathrm{~mA}$, 扫描范围为 $2 \theta=10^{\circ} \sim 90^{\circ}$, 扫描速度 $8 \% \mathrm{~min}$, 步宽为 $0.02 \%$ 。采用美国 FEI 公司场发射扫 描电镜(FEI Quanta 650)进行材料形貌表征。用 STA449F3 型热重 - 差热分析仪 (DSC/TG) 以 $10^{\circ} \mathrm{C} / \mathrm{min}$ 的升温速率升至 $1000^{\circ} \mathrm{C}$, 进行材料的热效 应分析。QuadraSorb SI 比表面分析仪(美国康塔仪 器公司)。

\section{3 电池组装及电化学性能测试}

用 $\mathrm{N}$-甲基吡咯烷酮(NMP)为溶剂，把制备的硅 基活性材料、导电碳黑(Super P) (40 nm, Timical)和 聚偏氟乙烯(PVDF)以 $8: 1: 1$ 的质量比混合搅拌成 浆料, 涂布于铜䈃上, 将涂好的极片转移到 $80^{\circ} \mathrm{C}$ 的 真空烘箱中烘干经成型压片。在充 $\mathrm{Ar}$ 手套箱 (Universal (2400/750/900), Mikrouna)中进行电池组 装。制备的硅基材料作为半电池正极, 锂片为负极, 以溶有 $2 \% \mathrm{VC}$ (碳酸乙烯酯)的 $1 \mathrm{~mol} / \mathrm{L} \mathrm{LiPF}_{6}$ 碳酸乙 烯酯/碳酸二甲酯 $(\mathrm{EC} / \mathrm{DMC}(v / v)=1: 1)$ 溶液为电解 液, 组装成 CR2025 型纽扣电池进行电化学测试。 使用电池测试仪(LAND 2001A, 武汉金诺电子有限 公司)对电池进行充放电测试, 充放电电压区间设为 $0.01 \sim 2.0 \mathrm{~V}$ 。

\section{2 结果与讨论}

\subsection{XRD 分析}

图 1 给出 SBA-15 镁热还原样品的 XRD 图谱。 图 1(a)是 400、500、600、660 ${ }^{\circ} \mathrm{C}$ 保温 $5 \mathrm{~h}$ 反应产物 

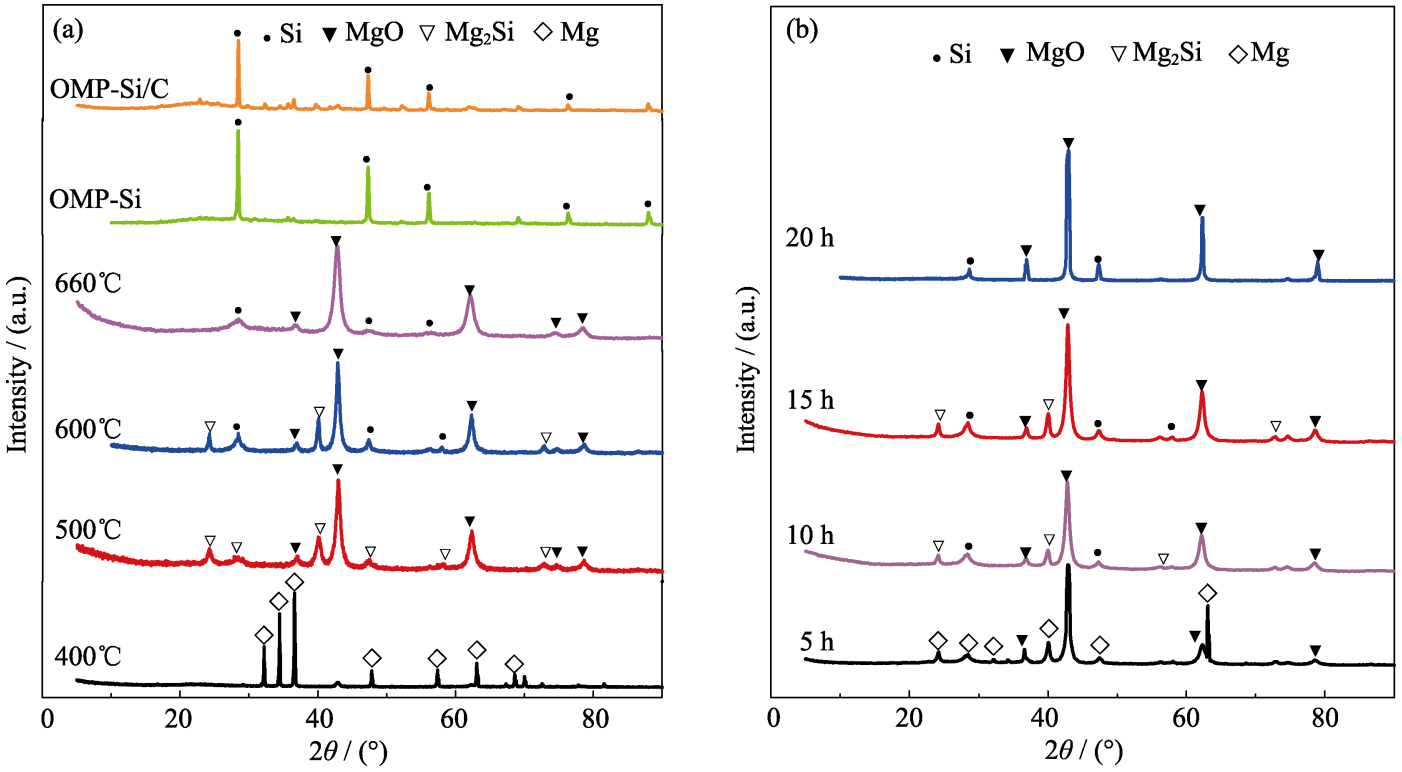

图 1 SBA-15 镁热还原样品的 XRD 图谱

Fig. 1 XRD patterns of the samples prepared by magnesiothermic reduction routes (a) Reaction at 400, 500, 600 and $660^{\circ} \mathrm{C}$ for $5 \mathrm{~h}, \mathrm{OMP}-\mathrm{Si}$ and OMP-Si/C; (b) Reaction at $560^{\circ} \mathrm{C}$ for $5,10,15$ and $20 \mathrm{~h}$

及样品 OMP-Si、OMP-Si/C 的 XRD 图谱, 而图 1(b) 则是 $560^{\circ} \mathrm{C}$ 保温 $5 、 10 、 15 、 20 \mathrm{~h}$ 反应产物的 XRD 图谱。图 1(a)中 $400^{\circ} \mathrm{C}$ 产物仅观察到 $\mathrm{Mg}$ 的衍射峰, 说明反应还未发生; $500^{\circ} \mathrm{C}$ 产物中出现 $\mathrm{MgO}$ 和 $\mathrm{Mg}_{2} \mathrm{Si}$ 的衍射峰; 当反应温度升高到 $600^{\circ} \mathrm{C}$ 时, 反应产物 中不仅有 $\mathrm{MgO}$ 和 $\mathrm{Mg}_{2} \mathrm{Si}$ ，且 $\mathrm{Si}$ 衍射峰出现; $660^{\circ} \mathrm{C}$ 产 物中 $\mathrm{Si}$ 和 $\mathrm{MgO}$ 的衍射峰强度增加, 而 $\mathrm{Mg}_{2} \mathrm{Si}$ 相则 完全消失。即保温时间一定, 随反应温度升高, 含 $\mathrm{Si}$ 相按 $\mathrm{Mg} \rightarrow \mathrm{Mg}_{2} \mathrm{Si} \rightarrow \mathrm{Mg}_{2} \mathrm{Si}+\mathrm{Si} \rightarrow \mathrm{Si}$ 顺序变化, 说明 镁热过程中先生成 $\mathrm{Mg}_{2} \mathrm{Si}$ 中间相, 随温度升高该相 逐渐转变为 $\mathrm{Si}$ 相。图 1(b)也展示出相类似的转变规 律, 随反应时间的延长, 含 $\mathrm{Si}$ 相仍按 $\mathrm{Mg} \rightarrow \mathrm{Mg}_{2} \mathrm{Si} \rightarrow$ $\mathrm{Mg}_{2} \mathrm{Si}+\mathrm{Si} \rightarrow \mathrm{Si}$ 顺序变化。还原样品进行酸洗及包碳 处理后的 XRD 图谱如图 1(a)所示, OMP-Si 和 OMP-Si/C 均在 $2 \theta=28.6^{\circ}$ 处观察到 $\mathrm{Si}$ 的主峰, 未出 现 $\mathrm{MgO} 、 \mathrm{Mg}_{2} \mathrm{Si}$ 等杂质, 说明经 $\mathrm{HCl}$ 处理后杂质被 去除, 得到纯硅相。经碳包覆后, 样品衍射峰并无明 显变化, 且在 $2 \theta=23^{\circ}$ 处出现无定形碳峰包。

从 $\mathrm{Mg}_{2} \mathrm{Si}$ 中间相存在及演化规律, 综合相关热 力学分析, 给出如图 2 所示反应温度-时间相图( $T-t$ 相图)。 $T-t$ 平面被中间相产生线( I )、转变结束线 (III)、 $\mathrm{Si}$ 相转变线( II )分割成以下四个相区: 未反应 区、 $\mathrm{Mg}_{2} \mathrm{Si}$ 中间相区、 $\mathrm{Mg}_{2} \mathrm{Si}+\mathrm{Si}$ 混合相区，以及 $\mathrm{Si}$ 相区。图中两垂直相交线即表明无论恒温反应抑或 定时保温, $\mathrm{Si}$ 相态转变均按 $\mathrm{Mg} \rightarrow \mathrm{Mg}_{2} \mathrm{Si} \rightarrow \mathrm{Mg}_{2} \mathrm{Si}+$ $\mathrm{Si} \rightarrow \mathrm{Si}$ 顺序。物系连续加热时亦按上述相变顺序 (见图中连续转变线), 快速升温时转变迅速完成且 相变温度高, 反之则转变温度低耗时长, 且常会出

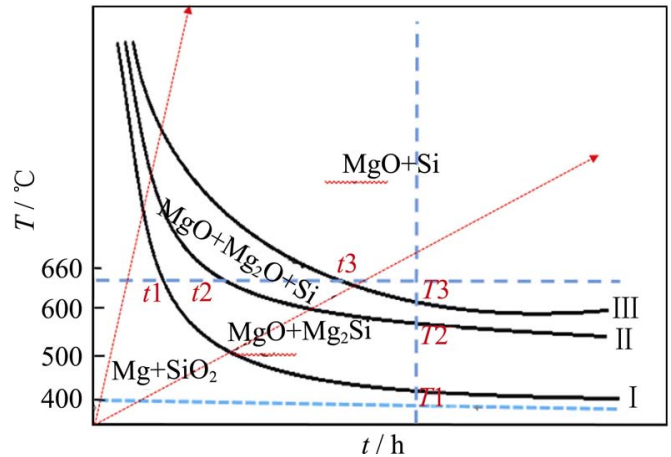

图 2 SBA-15 镁热还原反应相变温度-时间图( $T-t$ 图)

Fig. 2 Temperature-time graph reaction transformation of SBA-15 via magnesiothermic reduction routes ( $T-t$ graph)

现未转变残余相。综合图 1, 2 规律分析, $\mathrm{SiO}_{2}$ 镁热 还原相变经历下述反应过程:

$$
\begin{gathered}
2 \mathrm{Mg}(\mathrm{g})+\mathrm{SiO}_{2}(\mathrm{~s}) \rightarrow 2 \mathrm{MgO}(\mathrm{s})+\mathrm{Si}(\mathrm{s}) \\
2 \mathrm{Mg}(\mathrm{g})+\mathrm{Si}(\mathrm{s}) \rightarrow \mathrm{Mg}_{2} \mathrm{Si}(\mathrm{s})
\end{gathered}
$$

反应(1)与(2)联合得到反应(3), 而中间相 $\mathrm{Mg}_{2} \mathrm{Si}$ 存在对 $\mathrm{SiO}_{2}$ 的还原作用(反应(4)):

$$
\begin{aligned}
& 4 \mathrm{Mg}(\mathrm{g})+\mathrm{SiO}_{2} \rightarrow \mathrm{Mg}_{2} \mathrm{Si}(\mathrm{s})+2 \mathrm{MgO}(\mathrm{s}) \\
& \mathrm{Mg}_{2} \mathrm{Si}(\mathrm{s})+\mathrm{SiO}_{2}(\mathrm{~s}) \rightarrow 2 \mathrm{MgO}(\mathrm{s})+2 \mathrm{Si}(\mathrm{s})
\end{aligned}
$$

反应(3)、(4)揭示 $\mathrm{Mg}_{2} \mathrm{Si}$ 相过渡路径: 反应前期 $\mathrm{Mg}_{2} \mathrm{Si}$ 由反应界面处新生游离 $\mathrm{Si}$ 原子与后至 $\mathrm{Mg}$ 原 子化合而成, 因而前沿处新生 Si 迅速被耗尽, 故前 期仅存 $\mathrm{Mg}_{2} \mathrm{Si}$ 相; 反应中后期, 镁被消耗殆尽, 反 应区积累的 $\mathrm{Mg}_{2} \mathrm{Si}$ 相与液相区域中游离的 $\mathrm{SiO}_{2}$ 反应, 逐渐转化为 $\mathrm{Si}$ 晶相, 亦证明还原后期出现的 $\mathrm{Si}$ 相是 $\mathrm{SiO}_{2}$ 被还原而成, 并非前期残留。热力学分析发现, 即使超过 $1000^{\circ} \mathrm{C}$ 高温, 该反应亦可发生 ${ }^{[25-26]}$, 本工 
作在 $660^{\circ} \mathrm{C}$ 还原处理 $5 \mathrm{~h}$ 即可获得 $\mathrm{Mg}_{2} \mathrm{Si}$ 几近完全转 化的高 Si 收率。镁热还原 $\mathrm{SiO}_{2}$ 因其原始纳米结构 的不同、混料均匀性及加热制度等方面的影响, 其反 应动力学路径、产物组成及空间分布均存在差异 ${ }^{[27]}$ 。 本文所提出的 $\mathrm{Mg}_{2} \mathrm{Si}$ 中间相反应路径模型虽是基于 SBA-15 有序介孔组织结构, 但该模型亦可用于分 析其它镁还原反应路径。

\subsection{FESEM 照片}

通过场发射扫描电镜(FESEM)对样品的微观结 构和表面形貌进行表征。图 3(a) (b)观察到 SBA-15 由 $300 \mathrm{~nm} \times 600 \mathrm{~nm}$ 短柱状“藕根节”单元杂乱无规堆 积而成, 节单元(二维六方有序介孔组织)有团聚现 象。图 3(c) (d)发现 $400^{\circ} \mathrm{C}$ 反应 $20 \mathrm{~h}$ 产物与 SBA-15 原始形貌并无区别, 结合 XRD 结果说明此条件下 还原反应并未发生。图 3(e) (f) 是经 $500^{\circ} \mathrm{C}$ 反应 $20 \mathrm{~h}$ 产物的图像, 节单元表面变得粗糙, 少部分节单元 首尾相连形成几微米长的“藕根节”链, 但整体上仍 杂乱无序堆积。反应温度继续升高至 $600^{\circ} \mathrm{C}$, 如图 3(g) (h) 原本杂乱无序的单元节已经基本有序化排列, 节与节之间连接紧密。图 3(i) (j)还原条件为 $660^{\circ} \mathrm{C}$ 反 应 $5 \mathrm{~h}$, 从图 3(i)观察到全部节单元均链接, 并排形 成长 20 50 $\mu \mathrm{m}$ 的束捆状结构; 束捆内单元密切融 接成藕根链, 链间同向并排成束㧽系(图 3(j))。从图 $3(\mathrm{k})$ 观察到 $660^{\circ} \mathrm{C}$ 反应 $5 \mathrm{~h}$ 还原产物经盐酸浸蚀后除
去 $\mathrm{MgO} / \mathrm{Mg}_{2} \mathrm{Si}$ ，链束状结构并没有被破坏，说明束 捆内单元节纵向和侧向连接界面区域已反应焊合, 经 Si 晶相连接成不易崩散的结构。图 3(l)为酸浸样 品局部放大图像, 表面存在大量介孔道, 为 $\mathrm{Li}^{+}$迅捷 输运至 Si 介孔壁提供通道。

图 4 是 SBA-15/Mg 质量比 $1: 1$ 混合粉体的 $\mathrm{DSC} / \mathrm{TG}$ 分析曲线, 从图中发现以 $10^{\circ} \mathrm{C} / \mathrm{min}$ 连续升 温至 $620.8^{\circ} \mathrm{C}$ 处出现一个尖锐的放热峰并伴随着 $0.07 \%$ 的失重，即此时还原反应已发生。同时热量回 传致使 $\mathrm{Mg}$ 颗粒熔化, 因而镁熔点 $\left(650^{\circ} \mathrm{C}\right)$ 处无吸热 峰出现, 表明镁热还原反应与镁熔化在同一温度下 联动发生。从图 2 的结果可知, 升温速率较低还原 相变温度降低, 因而本研究所用 $4^{\circ} \mathrm{C} / \mathrm{min}$ 升温速率 样品在 $500^{\circ} \mathrm{C}$ 及以上温度均发生联动反应, 即所涉 体系属液/固还原反应。在上述反应一熔化联动机制 所致的液/固还原体制下，初始无规堆垛的 SBA-15 单元节出现纵横二维有序重构趋势。然而, Fan ${ }^{[28-29]}$ 及 $\mathrm{Bao}^{[21]}$ 对 $\mathrm{SiO}_{2}$ 气相镁热还原研究均发现, 反应后 的介孔硅仍保持杂乱无序堆积组织, 因此本研究出 现的二维有序重构现象可归因于液-固反应所致。

图 5 给出二维定向链束捆组织镁液微流场装 配模型示意图: (1) SBA-15/Mg 粉体混合物反应开 始随即推动 $M g$ 熔融, 直至 SBA-15 完全浸润悬浮 于连续镁熔体中; (2)SBA-15 反应界面附近温度高
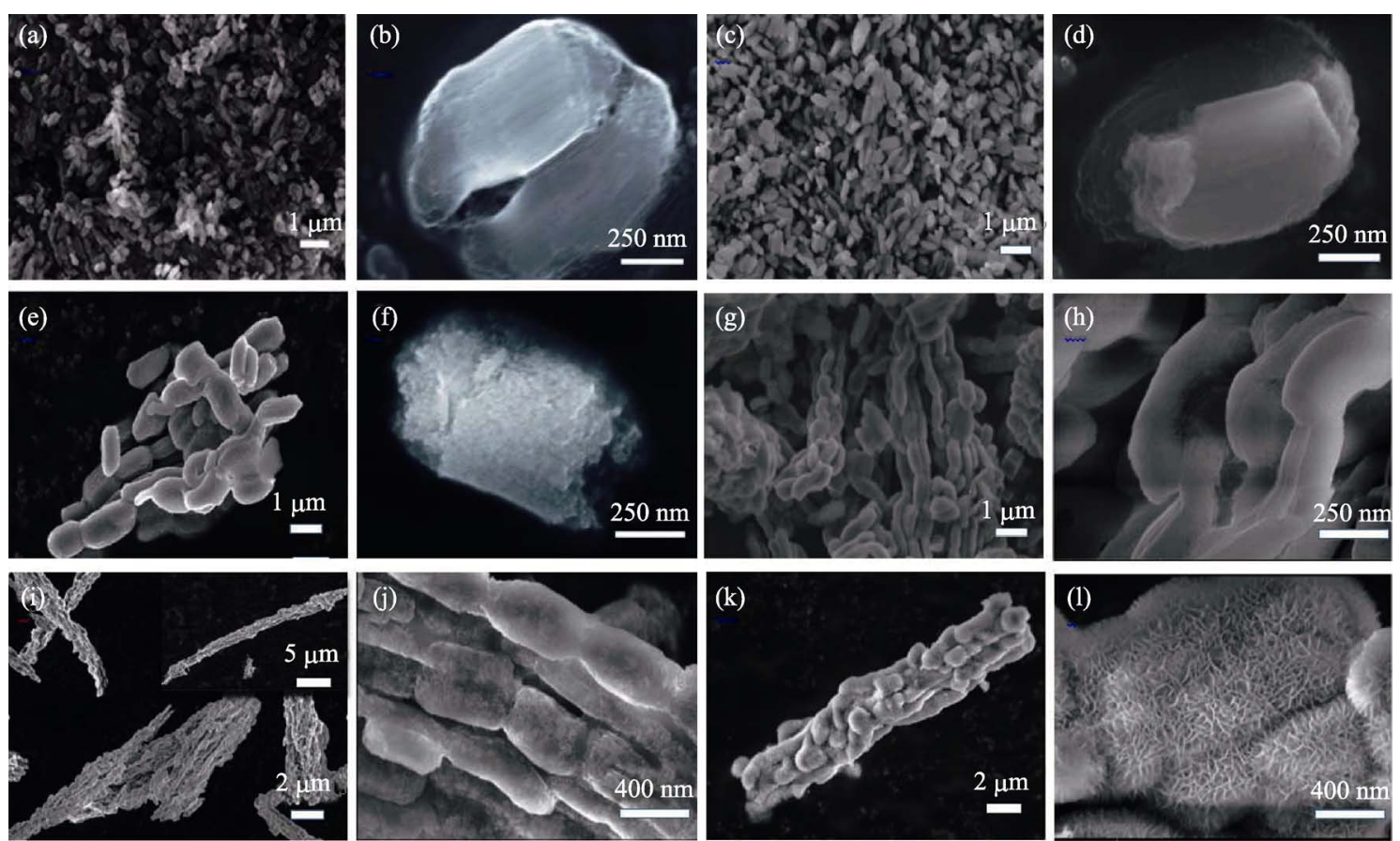
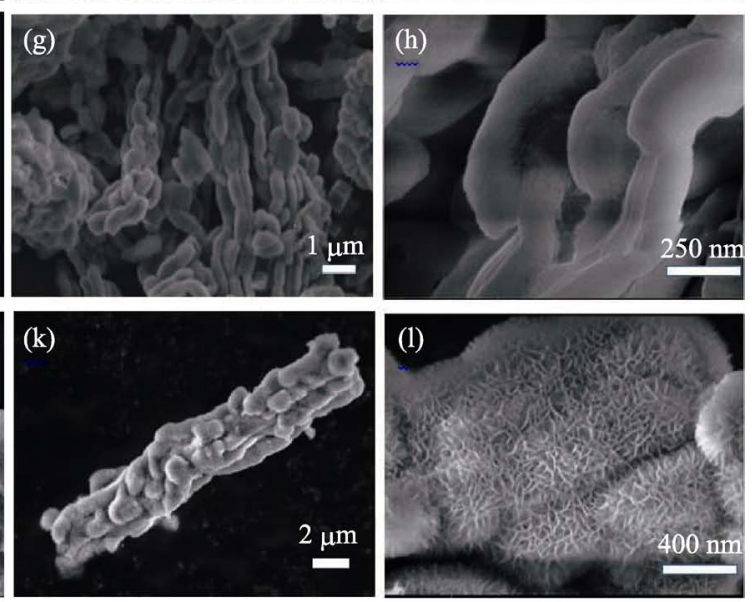

图 3 不同条件下合成的多孔硅 FESEM 照片

Fig. 3 FESEM images of porous Si synthesized under different conditions

(a, b) SBA-15; (c, d) Without acid etching, $400^{\circ} \mathrm{C}, 20 \mathrm{~h}$; (e, f) Without acid etching, $500^{\circ} \mathrm{C}, 20 \mathrm{~h}$; (g, h) Without acid etching, $600^{\circ} \mathrm{C}, 5 \mathrm{~h}$; (i, j) Without acid etching, $660^{\circ} \mathrm{C}, 5 \mathrm{~h}$; (k, l) Hydrochloric acid treatment OMP-Si, $660^{\circ} \mathrm{C}, 5 \mathrm{~h}$ 


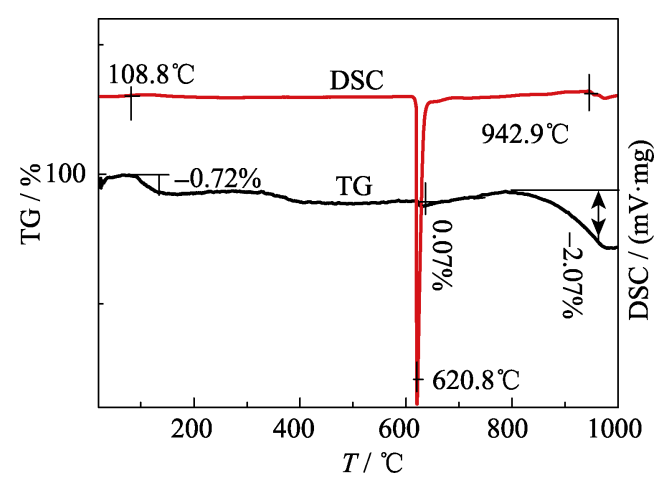

图 $4 \mathrm{SBA}-15 / \mathrm{Mg}$ 混合粉体 DSC/TG 分析曲线

Fig. 4 DSC/TG analyzing curves of SBA-15/Mg powdery admixture sample with weight ratio of $1: 1$ at temperature rise rate of $10^{\circ} \mathrm{C} / \mathrm{min}$

$\left(>3000^{\circ} \mathrm{C}\right)^{[30]}$, 与周围美液环境温度 $\left(500 \sim 660^{\circ} \mathrm{C}\right)$ 形 成超过 $2500^{\circ} \mathrm{C}$ 的温差。经估算该温度差在 $300 \mathrm{~nm}$ 宽的短柱节横向上形成 $10^{7{ }^{\circ}} \mathrm{C} / \mathrm{m}$ 量级的对流传热温 度梯度场, 导致柱横向出现镁液湍流浴(冷热液体 对流交换), 柱端面亦有; (3)湍流浴产生卷吸作用, 致使周围邻近短柱节纵向相吸形成“藕根节”链, 侧 向链间相吸归并成束，束进而凝并成捆。镁液微流 场对 SBA-15 的二维定向装配作用强弱与反应热效 应相关, 图 3 中 $500^{\circ} \mathrm{C}$ 和 $660^{\circ} \mathrm{C}$ 反应产物, 前者束捆 短细(仅几微米), 且排布有序程度低, 后者定向装 配作用强烈, 束捆粗且长达 30 50 $\mu \mathrm{m}$ 。本研究经镁 液微流场装配后原堆积松散的 SBA-15 粉体(振实密 度 $\left.0.1 \mathrm{~g} / \mathrm{cm}^{3}\right)$ 转向有序致密化堆积 $\left(0.8 \mathrm{~g} / \mathrm{cm}^{3}\right)$, 对各 种 Si 纳米结构固有的极低堆积密度问题是一重要
突破, 且装配电池的活性物质量以及储能密度也相 应大幅提升。

图 6 是 SBA-15 和 OMP-Si 样品的 $\mathrm{N}_{2}$ 吸附-解吸 等温线及孔径分布曲线。如图所示 $\mathrm{N}_{2}$ 吸附-解吸等 温线均属于IV型曲线, 表明 SBA-15 经镁热还原反 应后并未改变材料的介孔特性。基于非定域密度泛 函理论(NLDFT)模拟分析, SBA-15 还原成 OMP-Si 后, 总比表面积从 $543.49 \mathrm{~m}^{2} / \mathrm{g}$ 降至 $126.79 \mathrm{~m}^{2} / \mathrm{g}$; 介 孔比表面积从 375 降至 $124 \mathrm{~m}^{2} / \mathrm{g}$, 而微孔比表面从 $169 \mathrm{~m}^{2} / \mathrm{g}$ 迅速降至 $2 \mathrm{~m}^{2} / \mathrm{g}$ 。这种微孔、介孔急剧消 失的现象是由于镁热还原过程中 SBA-15 孔道不断 被熔融硅层填满, OMP-Si 表面逐渐平滑。孔径分析 结果显示 SBA-15、OMP-Si 孔径分别为 3.6 和 $5.7 \mathrm{~nm}$, 还原中由于 $\mathrm{MgO}$ 生长的随机性, 导致酸蚀后孔径不 同程度的扩大，但是仍能保持 SBA-15 的介孔特性。

\section{3 电化学性能测试分析}

图 7(a)为 OMP-Si/C 样品的前 10 次恒流充放电 循环电势-比容量曲线。初次放电曲线在 $0.9 \sim 0.2 \mathrm{~V}$ 处出现陡峭电压平台，与 SEI 膜的形成有关; $0.2 \sim 0.01 \mathrm{~V}$ 存在另一平缓电压平台, 为晶体 $\mathrm{Si}$ 与 $\mathrm{Li}$ 合金化并形成 $\mathrm{Li}_{x} \mathrm{Si}$ 非晶相过程 ${ }^{[31]}$; 在 $0.4 \mathrm{~V}$ 附近出 现一电压平台，与 $\mathrm{Li}_{x} \mathrm{Si}$ 非晶相脱 $\mathrm{Li}$ 去合金化过程 相关; 图中显示经最初几次循环后，放电曲线几近 重合，说明活性物质表面 SEI 膜趋于稳定，Si 介孔 结构芯/碳覆层/SEI 膜层复合组织的伸缩变形及破 坏程度亦趋于稳定 ${ }^{[32]}$ 。图 7(b)则给出 OMP-Si/C 随
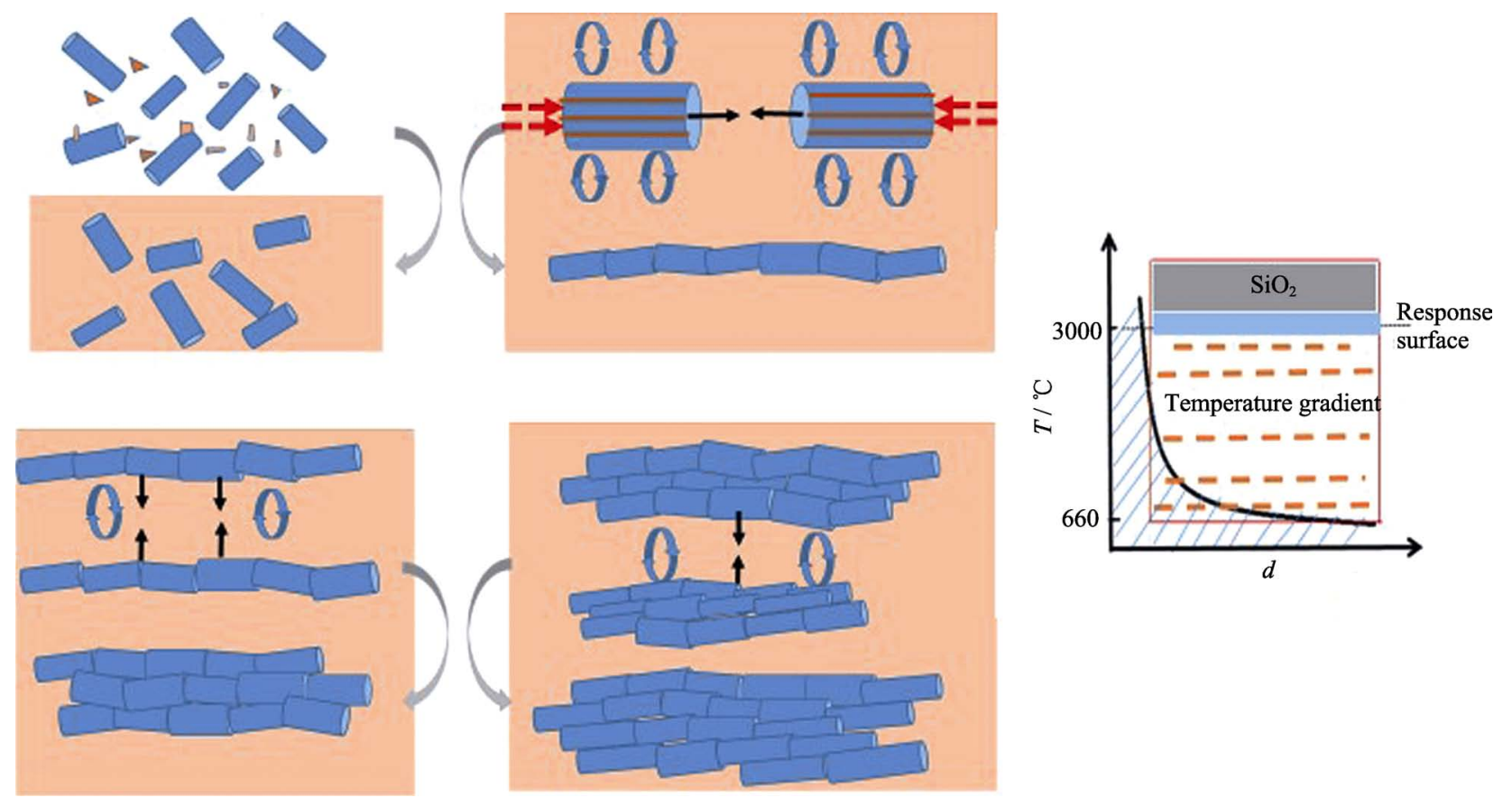

图 5 SBA-15 镁热还原反应机理示意图

Fig. 5 Schematic illustration of the mechanism of SBA-15 magnesiothermic reduction 


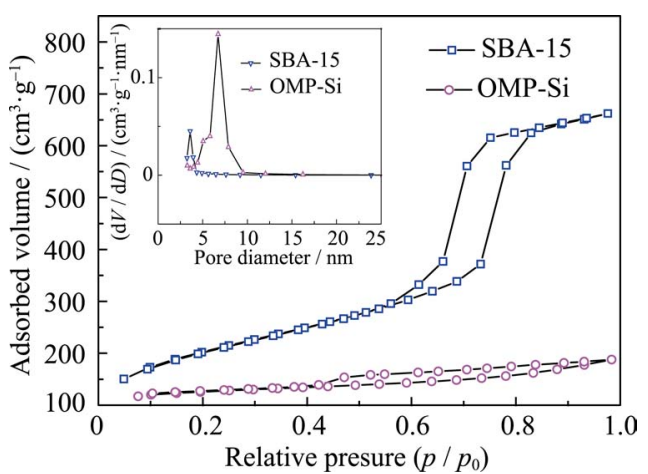

图 $6 \mathrm{~N}_{2}$ 吸附一解吸等温线和孔径分布图

Fig. $6 \mathrm{~N}_{2}$ adsorption and desorption isotherms with inset showing the pore size distribution curve for synthesized samples

循环次数放电容量、库仑效率变化曲线, OMP-Si 被列入以资比较。两者容量曲线趋势相似, 且 50 次循环相较第 5 次容量均衰减很少(OMP-Si/C: 840, $900 \mathrm{mAh} / \mathrm{g}$, OMP-Si: 330, $360 \mathrm{mAh} / \mathrm{g}$ ), 说明包碳层 并未改变有序介孔结构和机械强度, 保留原来介 孔组织缓冲体变效应特性的同时还显著提升了导 电性 ${ }^{[33]}$ 。

图 8 为有序介孔组织倍率性能测试结果。图 8(a), (b)分别为 OMP-Si 和 OMP-Si/C 介孔组织在不同电 流密度下循环容量变化。电流密度从 0.1 提高至 $2.0 \mathrm{~A} / \mathrm{g}$, 未包覆碳(OMP-Si)样品比容量仅减少 $1 / 3$,
而恢复至 0.1 时, 比容量基本恢复至最初水平; 结 合 SEM、BET 等分析结果说明, SBA-15 经二维定向 装配成束捆化有序介孔致密块体(30 50 $\mu \mathrm{m})$ 组织介 孔通道仍然高效畅通, $\mathrm{Li}^{+}$输运不受阻碍, 仍具备良 好的倍率特性。而经碳包覆后(见图 8(b))OMP-Si/C 倍率性能有所劣化, 碳包覆层虽然强化了导电网络, 但原 $\mathrm{Si}$ 介孔通道内部和孔口尺寸减小致使 $\mathrm{Li}^{+}$输运 受到一定迟滞, 导电体系组构方式仍然存在挑战。

\section{3 结论}

以 SBA-15 为前驱体, 经过镁热还原和包覆碳 层修饰技术路线, 成功制备出高储能密度的二维有 序束捆状介孔硅/碳复合结构。在 SBA-15 镁热还原 过程中存在 $\mathrm{Mg}_{2} \mathrm{Si}$ 中间相反应路径, 并藉此提出 $T-t$ 转变图。镁热还原反应过程中, 反应一熔化两过 程协调联动发生, 低于 $\mathrm{Mg}$ 熔点处即可发生液/固还 原反应。温度梯度差导致反应区周围存在镁液湍流 浴, 致使短柱单元纵向链接、侧向归束、束间凝并 成束捆, 即通过微流场二维定向装配机制堆砌出致 密有序介孔 $\mathrm{Si}$ 结构, 且该组织能有效削减充放电过 程中体积变化, 展现出优异的循环稳定性能和倍率 性能。
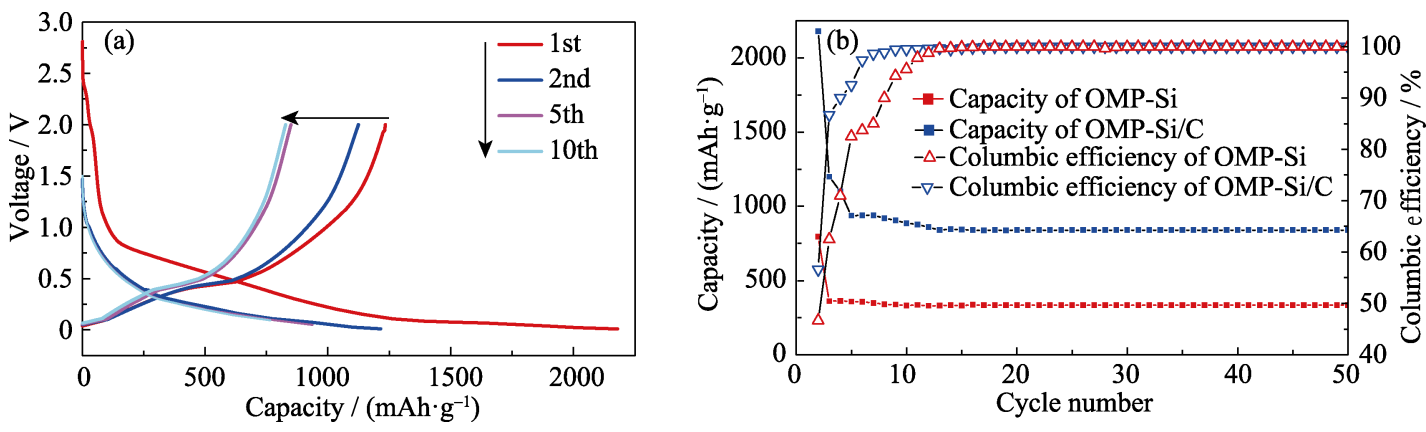

图 7 OMP-Si/C 样品的充放电曲线(a), 比容量和库仑效率(b)

Fig. 7 (a) Charge/discharge curves of OMP-Si/C sample and (b) the specific capacity and columbic efficiency versus cycle number for as-prepared samples
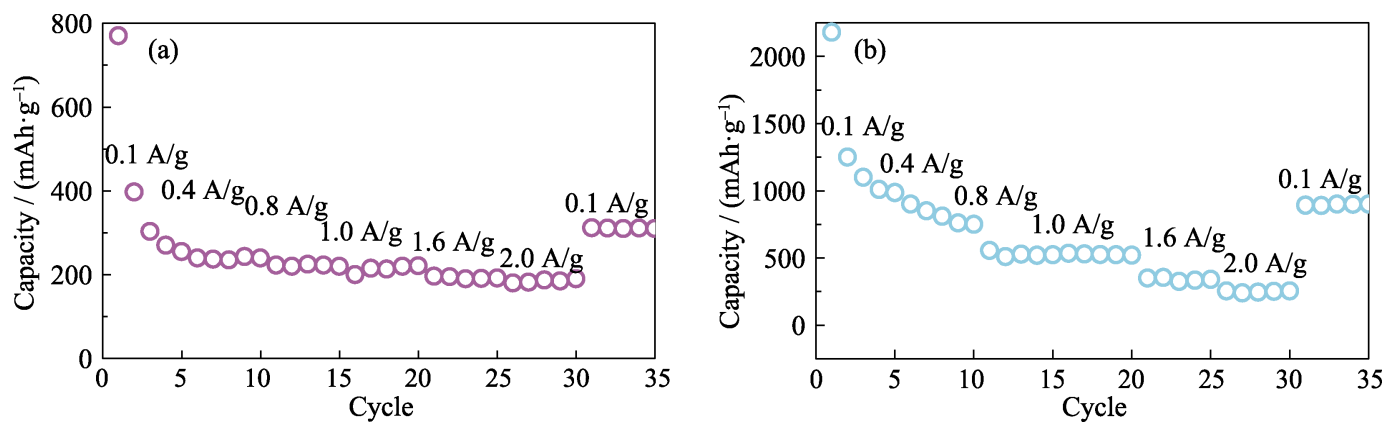

图 8 (a) OMP-Si 样品的倍率性能和(b) OMP-Si/C 样品的倍率性能

Fig. 8 Rate performance of (a) OMP-Si and (b) OMP-Si/C 


\section{参考文献:}

[1] ARMAND M, TARASCON J M. Building better batteries. Nature, 2002, 452(7179): 652-657.

[2] TERADA N, YANAGI T, ARAI S, et al. Development of lithium batteries for energy storage and EV applications. Journal of Power Sources, 2001, 100: 80-92.

[3] SCROSATI B. Technology: charging towards the superbattery. Nature, 2011, 473: 448-449.

[4] WINTER M, BESENHARD J O, SPAHR M E, et al. Insertion electrode materials for rechargeable lithium batteries. Adv. Mater., 1998, 10(10): 725-763.

[5] BOUKAMP B A, LEASH G C, HUGGINS R A J. All solid lithium electrodes with mixed conductor matrix. Electrochem. Soc., 1981, 128: $725-729$.

[6] WU H, CUI Y. Designing nanostructured Si anodes for high energy lithium ion batteries. Nano Today, 2012, 7: 414-429.

[7] CUI L F, RUFFO R, CHAN C K, et al. Crystalline-amorphous core-shell silicon nanowires for high capacity and high current battery electrodes. Nano Lett., 2009, 9(1): 491-495.

[8] ZHOU Y L, JIANG X L, CHEN L, et al. Novel mesoporous silicon nanorod as an anode material for lithium ion batteries. Electrochim. Acta, 2014, 127: 252-258.

[9] YAO Y, MCDOWELL M T, RYU I, et al. Interconnected silicon hollow nanospheres for lithium-ion battery anodes with long cycle life. Nano Lett., 2011, 11(7): 2949-2954.

[10] DATTA M K, MARANCHI J, CHUNG S J, et al . Amorphous silicon-carbon based nano-scale thin film anode materials for lithium ion batteries. Electrochim. Acta, 2011, 56: 4717-4723.

[11] TANG Y Y, XIA X H, YU Y X, et al. Cobalt nanomountain array supported silicon film anode for high-performance lithium ion batteries. Electrochim. Acta , 2013, 88: 664-670.

[12] HUANG Y, DUAN X F, WEI Q Q, et al. Directed assembly of one-dimensional nanostructures into functional networks. Science, 2001, 291(5504): 630-633.

[13] ZHONG H, ZHAN H, ZHOU Y H. Synthesis of nanosized mesoporous silicon by magnesium-thermal method used as anode material for lithium ion battery. Journal of Power Sources, 2014, 262: 10-14.

[14] BANG B M, LEE J I, KIM H, et al. High-performance macroporous bulk silicon anodes synthesized by template-free chemical etching. Advanced Energy Materials, 2012, 2: 878-883.

[15] LIANG J W, LI X N, HOU Z G, et al. A deep reduction and partial oxidation strategy for fabrication of mesoporous $\mathrm{Si}$ anode for lithium ion batteries. ACS Nano, 2016, 10(2): 2295-2304.

[16] SUN C F, ZHU H, OKADA M, et al. Interfacial oxygen stabilizes composite silicon anodes. Nano Lett., 2015, 15(1): 703-708.

[17] ZHANG R Y, DU Y J, SHEN D, et al. Highly reversible and large lithium storage in mesoporous $\mathrm{Si} / \mathrm{C}$ nanocomposite anodes with silicon nanoparticles embedded in a carbon framework. Adv. Mater.
2014, 26: 6749-6755.

[18] TARASCON J M. Key challenges in future Li-battery research. Phil. Trans. R. Soc. A, 2010, 368: 3227-3241.

[19] GAO P C, SIMON P, FAVIER F. Silicon carbide with tunable ordered mesoporosity. Microporous and Mesoporous Materials, 2013, 180: $172-177$.

[20] HONG I, CROSATI B, CROCE F. Mesoporous, Si/C composite anode for Li battery obtained by 'magnesium-thermal' reduction process. Solid State Ionics, 2013, 232: 24-28.

[21] BAO Z H, WEATHERSPOON M R, SHIAN S, et al. Chemical reduction of three-dimensional silica micro-assemblies into microporous silicon replicas. Nature, 2007, 446: 172-175.

[22] JIA H P, GAO P F, YANG J, et al. Novel three-dimensional mesoporous silicon for high power lithium-ion battery anode material. Advanced Energy Materials, 2011, 1: 1036-1039.

[23] LIU N A, HUO K F, MCDOWELL M T, et al. Rice husks as a sustainable source of nanostructured silicon for high performance Li-ion battery anodes. Scientfic Reports, 2013, 3: 1919-1-7.

[24] TANG Y P, YUAN S, GUO Y Z, et al. Highly ordered mesoporous $\mathrm{Si} / \mathrm{C}$ nanocomposite as high performance anode material for Li-ion batteries. Electrochim. Acta, 2016, 200: 182-188.

[25] XIAO C M, DU N, SHI X X, et al. Large-scale synthesis of Si@C three-dimensional porous structures as high-performance anode materials for lithium-ion batteries. Journal of Materials Chemistry, 2014, 2: 20494-20499.

[26] KIM W S, HUA Y, SHIN J H, et al. Scalable synthesis of silicon nanosheets from sand as an anode for Li-ion batteries. Nanoscale, 2014, 6: 4297-4302.

[27] AHN JIHON, KIM HEE SOO, PYO JUNG, et al. Variation in crystalline phases: controlling the selectivity between silicon and silicon carbide via magnesiothermic reduction using silica/carbon composites. Chemistry of Materials, 2016, 28: 1526-1536.

[28] TAO H C, FAN L Z, QU X H. Facile synthesis of ordered porous Si@C nanorods as anode materials for Li-ion batteries. Electrochim. Acta, 2012, 71: 194-200.

[29] WANG L L, MUNIR Z A, MAXIMOV Y M. Thermite reactions: their utilization in the synthesis and processing of materials. $J$. Mater. Sci., 1993, 28: 3693-3708.

[30] HUGGINS R A. Lithium alloy negative electrodes. Power Sources, 1999, 81-82: 13-19.

[31] YU X L, YANG J, FENG X J, et al. Preparation and electrochemical properties of porous silicon/carbon composite as negative electrode materials. Journal of Inorganic Materials, 2013, 28(9): 937-942.

[32] WADA T, ICHITSUBO T, YUBUTA K, et al. Buk-nanoporousilicon negative electrode with extremely high cyclability for lithium-ion batteries prepared using a top-down process. Nano Lett., 2014, 14: 4505-4510.

[33] KIM H, CHOU C Y, EKERDT J G, et al. Structure and properties of Li-Si alloys: a first-principles study. Journal of Physical Chemistry, 2011, 115(5): 2514-2521. 